\title{
PORTA-ENXERTOS PARA A LIMA-ÁCIDA-'TAHITI' NA REGIÃO DE BEBEDOURO, SP. ${ }^{1}$
}

\author{
JOSÉ ORLANDO DE FIGUEIREDO ${ }^{2,3}$, EDUARDO SANCHES STUCHI ${ }^{4}$, LUIZ CARLOS DONADIO ${ }^{3,4,5}$, \\ JOAQUIM TEÓFILO SOBRINHO ${ }^{2,3}$, FRANCISCO FERRAZ LARANJEIRA ${ }^{6}$, ROSE MARY PIO ${ }^{2,3}$ e \\ OTÁVIO RICARDO SEMPIONATO ${ }^{4}$
}

\begin{abstract}
RESUMO - Foi instalado um experimento de seleção de porta-enxertos para a lima-ácida-'Tahiti' (Citrus latifolia Tanaka), em dezembro de 1988, na Estação Experimental de Citricultura de Bebedouro-SP, com o objetivo de conhecer seu comportamento e oferecer novas opções de plantio para as condições ecológicas semelhantes às daquela região. A variedade copa, originária do BAG-Citros do IAC, localizado no Centro de Citricultura Sylvio Moreira, Cordeirópolis-SP, é um clone nucelar de 'Tahiti', denominado IAC-5. Os portaenxertos, que tiveram a mesma origem, foram: tangerinas-'Sunki' (Citrus sunki Hort. ex Tanaka); 'Cleópatra' (Citrus reshni Hort. ex Tan.); 'Batangas' e 'Oneco' (Citrus reticulata Blanco); trifoliata-EEL (Poncirus trifoliata Raf.); limão- 'Cravo' (Citrus limonia Osbeck); limão-'Volkameriano Catania 2' (Citrus volkameriana Tan. \& Pasq.); tangelo-'Orlando' (C. reticulata Blanco x Citrus paradisi Macf.); citrumelo- 'Swingle' (P. trifoliata Raf. X C.paradisi Macf.); citrange-'Morton' (P. trifoliata Raf. X C. sinensis (L.) Osbeck) e laranja'Caipira DAC' (C. sinensis (L.) Osbeck). Com relação à produção, avaliada no período de 1991 a 1998, os porta-enxertos de melhor comportamento foram o tangelo-'Orlando', citrange-'Morton' e citrumelo-'Swingle'. As mais baixas produções ocorreram nos portaenxertos de tangerina e de laranja- 'Caipira DAC'. O limão- 'Cravo' apresentou produção intermediária e proporcionou curta vida útil às plantas.
\end{abstract}

Termos para indexação : seleção, Citrus latifolia, produção, citros .

\section{ROOTSTOCKS FOR 'TAHITI' ACID LIME IN BEBEDOURO REGION, SP, BRAZIL}

\begin{abstract}
In 1988 an experiment was established in Bebedouro, SP (Brazil), in order to evaluate eleven rootstocks for 'Tahiti' lime (Citrus latifolia Tanaka). Scion variety was the IAC 5 cultivar, from IAC germplasm bank at Cordeirópolis, SP; all rootstocks were also obtained at that institution. A randomized blocks design was used as statistical model, with eleven blocks and one plant per treatment. The eleven treatments were: 'Sunki' (Citrus sunki Hort. ex Tanaka), 'Cleópatra'(Citrus reshni Hort. ex Tan.), 'Batangas' and 'Oneco' (Citrus reticulata Blanco) mandarins; EEL trifoliate (Poncirus trifoliata Raf.); Rangpur lime (Citrus limonia Osbeck); 'Catania 2 Volkamer' lemon (Citrus volkameriana Tan. \& Pasq.); 'Orlando' tangelo (C. reticulata Blanco x C. paradisi Macf.); 'Swingle' citrumelo (P. trifoliata Raf. X Citrus paradisi Macf.); 'Morton' citrange (P. trifoliata Raf. X C. sinensis (L.) Osbeck) and 'Caipira DAC' sweet orange (C. sinensis (L.) Osbeck). Yield was evaluated annually from 1991 to 1998; drought resistance was evaluated in 1991, 1994 and 1995; fruit features were assessed in 1997 and canopy height and diameter were measured in 2000. Highest mean yields were associated to 'Orlando' tangelo, 'Morton' citrange and 'Swingle' citrumelo rootstocks. The lowest yields were related to mandarins and 'Caipira DAC' sweet orange. Plants with 'Rangpur' lime as rootstock bore intermediate yields and their productive life was short.
\end{abstract}

Index terms: selection, Citrus latifolia, yield, citrus.

\section{INTRODUÇÃo}

A lima-ácida-‘Tahiti’ (Citrus latifolia Tanaka), popularmente conhecida no Brasil como um limão, atualmente vem despertando interesse para a ampliação dos plantios comerciais. Isso ocorre em função de seu bom comportamento diante das principais doenças e pragas que estão presentes nos pomares cítricos e que vêm causando grandes prejuízos para os produtores de laranjas doces.

Aliado a esta constatação, está o fato de que os europeus, acostumados a consumir os limões de casca amarela, estão aprendendo a consumir o 'Tahiti', de casca verde. Presentemente, já existe um lento, mas significativo incremento nas importações de frutos dessa espécie, principalmente pelos países integrantes do Mercado Comum Europeu. Essa abertura de mercado constituise no principal estímulo ao cultivo do 'Tahiti'. Por outro lado, as árvores de 'Tahiti' apresentam a marcante característica de florescimento continuado, com duas safras bem definidas: a principal no primeiro semestre (janeiro a junho), quando os preços são menos atraentes, e uma segunda colheita concentrada nos meses de julho a novembro, com preços mais elevados (Figueiredo et al.,1976). Assim, a estratégia para a comercialização

\footnotetext{
1 (Trabalho 239/2000). Recebido: 30/01/2000. Aceito para publicação: 18/01/2002.

2 Centro de Citricultura Sylvio Moreira /IAC. Caixa Postal 04. CEP 13.490-970. Cordeirópolis - SP.

3 Bolsista do CNPq

4 EECB, C.P. 49, CEP 14700-000, Bebedouro - SP

5 FCAV/UNESP, Rodovia Carlos Tonnani, km 5, CEP 14870-000, Jaboticabal - SP

6 EMBRAPA - Mandioca e Fruticultura, C. P. 7, CEP 44380-000 - Cruz das Almas - BA
} 
de seus frutos constitui-se em exportá-los e industrializá-los para escoamento da safra no primeiro semestre. No segundo semestre, quando a produção é menor e a demanda aumenta, o atendimento maior deve ser o mercado interno para consumo "in natura".

Em São Paulo, nos anos agrícolas 1996/97 e 1997/98, a produção de limas ácidas atingiu 28,26 e 31,82 milhões de caixas de $25,0 \mathrm{~kg}$, respectivamente. $\mathrm{O}$ valor desta produção foi estimado ao redor de 119 e 76 milhões de reais, respectivamente. Isto corresponde a $1,17 \%$ e 0,67 do valor de toda a atividade agropecuária do Estado de São Paulo, superando assim a cultura do amendoim, da melancia, da uva fina de mesa, do arroz em casca, da mandioca para mesa, do tomate para indústria e do trigo. Por esses números, o Estado de São Paulo é o grande produtor brasileiro de 'Tahiti', com cerca de $73 \%$ da safra brasileira (Donadelli et al., 2000).

Considerando o uso de porta-enxertos, verifica-se que há pequena quantidade de trabalhos já realizados e feitos em São Paulo para o 'Tahiti'. O uso de outras espécies que não o limão-'Cravo', porta-enxerto que predomina nos plantios do 'Tahiti', é importante e necessário, uma vez que os fungos do gênero Phytophthora encurtam a vida útil das plantas com aquele porta-enxerto. Assim, o objetivo deste trabalho foi avaliar portaenxertos que pudessem substituir o limão- 'Cravo' como portaenxerto para o 'Tahiti' nas condições edafoclimáticas de Bebedouro. Dados iniciais desse trabalho já foram apresentados de maneira preliminar por Figueiredo et al. (1996).

\section{MATERIAL E MÉTODOS}

O experimento foi conduzido na Estação Experimental de Citricultura de Bebedouro (EECB), no Município de BebedouroSP (Latitude $20^{\circ} 53^{\prime} 16^{\prime \prime}$ S, Longitude 48 28'11' W), altitude de $601 \mathrm{~m}$, em um solo classificado como Latossolo Vermelho-Escuro, epieutrófico, endoálico, A moderado, textura média, (Haplustox). O clima é do tipo Cwa (subtropical com inverno moderado e seco, verão quente e chuvoso), com temperatura média máxima de $28,8^{\circ} \mathrm{C}$, média mínima $18,3^{\circ} \mathrm{C}$ e temperatura média de $23,5^{\circ} \mathrm{C}$; a precipitação anual é de $1.522 \mathrm{~mm}$ (dados médios de 13 anos de observação no posto meteorológicos da EECB). Os dados de balanço hídrico em alguns anos do experimento estão no Quadro 1 .

A instalação do experimento foi em 07-12-1988, com 11 tratamentos, em espaçamento de $8 \mathrm{~m} \times 6 \mathrm{~m}$ e conduzido sem irrigação. A variedade copa proveio de um clone nucelar de 'Tahiti', denominado IAC-5, existente no Banco Ativo de Germoplasma de Citros localizado no Centro de Citricultura Sylvio Moreira, Cordeirópolis-SP. Esse material já fora testado anteriormente em um experimento de seis clones de limão-'Tahiti' e apresentou o melhor comportamento entre eles (Figueiredo et al., 1976). O material não apresenta hipertrofia dos cálices florais (Donadio \& Figueiredo, 1972) e é mais tolerante à tristeza que os outros clones estudados (Figueiredo et al., 1976; Rodriguez et al., 1973). As variedades porta-enxerto, também oriundas do BAG citros-IAC, foram as seguintes: tangerinas-'Sunki' (Citrus sunki Hort. ex Tanaka); 'Cleópatra' (Citrusreshni Hort. ex Tan.); 'Batangas' e 'Oneco' (Citrus reticulata Blanco); trifoliatas-EEL (Poncirus trifoliata Raf.); limão-'Cravo' (Citrus limonia Osbeck); limão-'Volkameriano Catania 2' (Citrus volkameriana Ten. \&
Pasq.); tangelo- 'Orlando' (Citrus reticulata Blanco x C. paradisi Macf.); citrumelo-'Swingle' (P. trifoliata Raf. x C. paradisi Macf.); citrange-'Morton' (P. trifoliata Raf. X C. sinensis (L.) Osbeck) e laranja-'Caipira DAC' (Citrus sinensis (L.) Osbeck). Os portaenxertos estudados são tolerantes ao vírus da tristeza. Foram utilizadas dez mudas uniformes para cada tratamento em parcelas de uma planta, em blocos ao acaso, havendo somente bordaduras externas. Devido à característica de a cultivar apresentar multiplicidade de floradas, as colheitas e pesagem da produção foram realizadas até quatro vezes por ano, sendo somados os resultados obtidos em cada ano.

Foram coletados os dados da produção das plantas, em peso de frutos, nos anos de 1991 a 1998, do desenvolvimento vegetativo das plantas (altura, diâmetro), no ano de 1998, de sua resistência à seca, em 1991, 1994 e 1995 e da qualidade dos frutos, em 2000. Os valores de produção anual foram somados para a obtenção da produção acumulada de frutos por planta no período. Os diâmetros médios das copas foram obtidos por meio de duas medições em eixos perpendiculares, situados na metade da altura das plantas, com uso de régua graduada. A altura das plantas foi medida desde o nível do solo até o topo das plantas. Com os dados de altura e diâmetro da copa, obtidos no ano de 1998, calculou-se o volume da copa através da fórmula: $V=2 / 3 \mathrm{PR}^{2} \mathrm{H}$, onde $\mathrm{V}$ representa o volume $\mathrm{em}^{3}$; $\mathrm{R}$, o raio médio; e $\mathrm{H}$, a sua altura (Mendel, 1956).

Os dados de resistência à seca foram obtidos por meio de uma escala de notas em avaliações visuais realizadas nos meses de setembro, nos anos de 1991, 1994 e 1995, anos estes de severo déficit hídrico (Quadro 1). Essa escala variou de um a três, sendo a nota 1 para o mais resistente (resistente), a nota 2 para resistência intermediária (intermediária) e a nota 3 para o menos resistente (sensível). As notas sempre foram atribuídas por dois observadores de forma independente e, posteriormente, calculadas as médias.

Em 1997, avaliou-se a influência dos porta-enxerto em três características físicas dos frutos; para isto, foram coletadas amostras de 10 frutos, em quatro das repetições. As amostras foram pesadas e calculou-se o peso médio (massa fresca) dos frutos em gramas. Os frutos de cada amostra foram medidos individualmente (altura e diâmetro) e calculou-se a média de cada uma das duas características.

Em 1998, realizou-se o levantamento do número de plantas mortas (falhas) por tratamento, sendo posteriormente calculado o percentual das mesmas em relação ao número inicial de plantas por tratamento, que era de 10.

Os dados de produção acumulada de frutos, de desenvolvimento vegetativo das plantas e de resistência à seca, em cada avaliação e na média do período, e daqueles referentes à qualidade dos frutos foram submetidos a análise de variância, e as médias comparadas pelo teste de Tukey, ao nível de 5\% de probabilidade de erro.

\section{RESULTADOS E DISCUSSÃO}

\section{Produção}

Pode-se notar que a melhor produtividade foi induzida pelo porta-enxerto tangelo-'Orlando' que diferiu de 'Cleópatra', 'Batangas', 'Oneco' e 'Caipira', que apresentaram a menor 
produtividade (Tabela 1). 'Morton', 'Swingle', 'Catania 2', 'EEL', 'Cravo' e 'Sunki' não diferiram entre si, nem de 'Orlando'. Notese que o tangelo- 'Orlando' apresentou esse desempenho, apesar de sofrer $40 \%$ de perdas de plantas devido à gomose de Phytophthora. Foi também o porta-enxerto mais sensível à seca, neste experimento, e também durante a forte estiagem do ano de 1994. Com relação à produção de frutos, de um modo geral, estes dados estão de acordo com os resultados iniciais obtidos do mesmo experimento (Figueiredo et al., 1996), para a maioria dos porta-enxertos. Fazem exceção o limão- 'Cravo' e o citrumelo'Swingle'. O limão-'Cravo' destacou-se no período inicial, mas foi apenas intermediário em produção no período total dos oito anos considerados. O citrumelo-'Swingle', que nos primeiros anos apresentou produções intermediárias, melhorou sua posição no total dos anos, colocando-se entre os três porta-enxertos mais produtivos, o que indica a sua boa adaptação às condições edafoclimáticas de Bebedouro. Outros porta-enxertos como 'Sunki', 'Batangas' e 'Caipira DAC' alteraram também sua posição, mas não apresentaram resultados de maior interesse.

\section{Qualidade dos frutos}

Quanto às características de qualidade estudadas, não houve diferenças significativas, em função dos porta-enxertos

TABELA 1 - Produção acumulada e produção média de limão-'Tahiti' (Kg.planta-1) enxertado sobre diversos porta-enxertos no período de 1991 a 1998, número de plantas remanescentes e porcentagem de perda de plantas em 1998. Bebedouro-SP, 2000.

\begin{tabular}{|c|c|c|c|c|}
\hline Porta-enxertos & $\begin{array}{c}\text { Produção } \\
\text { A cumulada } \\
\left(\text { kg.planta }{ }^{-1}\right)\end{array}$ & $\begin{array}{l}\text { Produção M édia no } \\
\text { período } 1991 \text { a } 1998 \\
\left(\text { kg.p lanta }{ }^{-1}\right)\end{array}$ & $\begin{array}{c}\text { Plantas } \\
\text { remanescentes em } \\
1998 \text { (número) }\end{array}$ & $\begin{array}{c}\text { Perda de p lantas } \\
(\%)\end{array}$ \\
\hline Citrange-'M orton' & $347,2 \mathrm{ab}$ & 43,4 & 10 & 0 \\
\hline Citrum elo-'Sw ingle' & $317,3 \mathrm{ab}$ & 39,7 & 10 & 0 \\
\hline Trifoliata-'EEL' & 281,9 abcd & 35,2 & 10 & 0 \\
\hline Limão-'Cravo' & $272,3 \mathrm{abcd}$ & 34,0 & 0 & 100 \\
\hline T angerina-'Sunki' & $216,9 \mathrm{abcd}$ & 27,1 & 9 & 10 \\
\hline $\mathrm{T}$ angerina-'C leóp atra' & $183,1 \mathrm{bcd}$ & 22,9 & 10 & 0 \\
\hline T angerin $\mathrm{a}-$ ' $^{\mathrm{B}}$ at angas' & $141,7 \mathrm{~cd}$ & 17,7 & 8 & 20 \\
\hline $\mathrm{CV}(\%)$ & 47,46 & - & - & \\
\hline
\end{tabular}

TABELA 2 - Valores médios de massa fresca, altura e diâmetro dos frutos de limão-'Tahiti' sobre 11 portaenxertos em 1997. Bebedouro-SP, 2000,

\begin{tabular}{lccc}
\hline Porta-enxertos & Massa Fresca & Altura & Diâmetro \\
\hline & $(\mathrm{g})$ & $(\mathrm{cm})$ & $(\mathrm{cm})$ \\
Tangelo-'Orlando' & $89 \mathrm{a}^{1}$ & $5,88 \mathrm{a}$ & $5,30 \mathrm{a}$ \\
Citrange-'M orton' & $91 \mathrm{a}$ & $5,96 \mathrm{a}$ & $5,35 \mathrm{a}$ \\
Citrumelo-'Swingle' & $95 \mathrm{a}$ & $6,00 \mathrm{a}$ & $5,39 \mathrm{a}$ \\
'Catania 2' & $89 \mathrm{a}$ & $5,91 \mathrm{a}$ & $5,14 \mathrm{a}$ \\
Trifoliata-'EEL' & $88 \mathrm{a}$ & $5,81 \mathrm{a}$ & $5,26 \mathrm{a}$ \\
Limão-'Cravo' & $-\mathrm{y}$ & $-\mathrm{y}$ & $-\mathrm{y}$ \\
Tangerina-'Sunki' & $90 \mathrm{a}$ & $6,01 \mathrm{a}$ & $5,26 \mathrm{a}$ \\
Tangerina-'Cleópatra' & $97 \mathrm{a}$ & $6,04 \mathrm{a}$ & $5,35 \mathrm{a}$ \\
Tangerina-'Batangas' & $81 \mathrm{a}$ & $5,81 \mathrm{a}$ & $5,46 \mathrm{a}$ \\
Tangerina-'Oneco' & $95 \mathrm{a}$ & $5,98 \mathrm{a}$ & $5,16 \mathrm{a}$ \\
Laranja-'Caipira DAC' & $-\mathrm{y}$ & $-\mathrm{y}$ & $-\mathrm{y}$ \\
\hline M édias & 91 & 5,93 & 5,30 \\
\hline DM S Tukey (5\%) & 29,06 & 0,63 & 0,50 \\
CV (\%) & 18,42 & 6,11 & 5,47 \\
\hline
\end{tabular}

Obs: médias seguidas de mesma letra minúscula na coluna não diferem entre si, pelo teste de Tukey $(\mathrm{p}<0,05)$

y tratamentos sem número de repetições para análise estatística
TABELA 3 - Resistência à seca do limão-'Tahiti' enxertado sobre 11 porta-enxertos em período de estiagem em 1991, 1994 e 1995, avaliada por notas $(1=$ resistente, $2=$ intermediário e 3 = sensível). Bebedouro-SP, 2000.

\begin{tabular}{llccc}
\hline \multicolumn{1}{c}{ Porta-enxertos } & \multicolumn{1}{c}{1991} & 1994 & 1995 & Média \\
\hline Limão-'Cravo' & $1,00 \mathrm{e}$ & $1,50 \mathrm{~d}$ & $1,40 \mathrm{c}$ & $1,30 \mathrm{~d}$ \\
'Catania 2' & $1,30 \mathrm{de}$ & $1,50 \mathrm{~d}$ & $1,30 \mathrm{c}$ & $1,37 \mathrm{~d}$ \\
Tangerina-'Sunki' & $2,10 \mathrm{abcd}$ & $1,80 \mathrm{~cd}$ & $1,90 \mathrm{bc}$ & $1,93 \mathrm{~cd}$ \\
Citrumelo-'Swingle' & $1,80 \mathrm{cde}$ & $2,20 \mathrm{bcd}$ & $2,70 \mathrm{a}$ & $2,23 \mathrm{bc}$ \\
Tangerina-'Cleópatra' & $2,20 \mathrm{abc}$ & $2,40 \mathrm{abc}$ & $2,50 \mathrm{ab}$ & $2,37 \mathrm{abc}$ \\
Citrange-'Morton' & $1,90 \mathrm{bcd}$ & $2,20 \mathrm{bcd}$ & $3,00 \mathrm{a}$ & $2,37 \mathrm{abc}$ \\
Trifoliata-'EEL' & $2,40 \mathrm{abc}$ & $2,50 \mathrm{abc}$ & $2,80 \mathrm{a}$ & $2,57 \mathrm{abc}$ \\
Laranja-'Caipira DAC' & $2,50 \mathrm{abc}$ & $2,60 \mathrm{ab}$ & $2,80 \mathrm{a}$ & $2,63 \mathrm{ab}$ \\
Tangerina-'Batangas' & $2,70 \mathrm{ab}$ & $2,80 \mathrm{ab}$ & $2,70 \mathrm{a}$ & $2,73 \mathrm{ab}$ \\
Tangerina-'Oneco' & $2,80 \mathrm{a}$ & $2,90 \mathrm{ab}$ & $2,80 \mathrm{a}$ & $2,83 \mathrm{ab}$ \\
Tangelo-'Orlando' & $2,80 \mathrm{a}$ & $3,00 \mathrm{a}$ & $3,00 \mathrm{a}$ & $2,93 \mathrm{a}$ \\
\hline Médias Anuais & 2,14 & 2,31 & 2,45 & 2,30 \\
\hline DMS Tukey (5\%) & 0,89 & 0,79 & 0,68 & 0,65 \\
CV (\%) & 28,30 & 23,16 & 18,88 & 9,53 \\
\hline
\end{tabular}

Obs: médias seguidas de letras iguais na coluna não diferem entre si, pelo teste de Tukey $(\mathrm{p}<0,05)$ 
TABELA 4 - Altura diâmetro médio e volume das copas do limão'Tahiti', enxertado sobre 11 porta-enxertos, e porcentagens de falhas em 1998 Bebedouro-SP, 2000.

\begin{tabular}{lccc}
\hline Porta-enxertos & Diâmetro & Altura & Volume \\
\hline & $(\mathrm{m})$ & $(\mathrm{m})$ & $\left(\mathrm{m}^{3}\right)$ \\
Volkameriano 'Catania 2' & $7,35 \mathrm{a}$ & $4,38 \mathrm{ab}$ & 123,83 \\
Citrange-'Morton' & $6,48 \mathrm{ab}$ & $4,15 \mathrm{abc}$ & 91,20 \\
Tangelo-'Orlando' & $6,40 \mathrm{ab}$ & $4,41 \mathrm{a}$ & 94,53 \\
Trifoliata-'EEL' & $6,25 \mathrm{ab}$ & $4,05 \mathrm{abc}$ & 82,79 \\
Limão-'Cravo' & $6,1^{*}$ & $4,0^{*}$ & $77,89^{*}$ \\
Citrumelo-'Swingle' & $5,86 \mathrm{~b}$ & $3,90 \mathrm{abc}$ & 70,09 \\
Tangerina-'Oneco' & $5,86 \mathrm{~b}$ & $4,02 \mathrm{abc}$ & 72,24 \\
Tangerina-'Cleópatra' & $5,65 \mathrm{~b}$ & $3,72 \mathrm{c}$ & 62,15 \\
Tangerina-'Batangas' & $5,38 \mathrm{~b}$ & $3,78 \mathrm{bc}$ & 57,26 \\
Tangerina-'Sunki' & $5,38 \mathrm{~b}$ & $3,65 \mathrm{c}$ & 55,29 \\
Laranja-'Caipira DAC' & $4,1^{*}$ & $2,8^{*}$ & $24,63 *$ \\
\hline Médias & 6,07 & 4,00 & 77,13 \\
\hline DMS Tukey (5\%) & 1,31 & 0,62 & - \\
CV (\%) & 11,41 & 8,19 & -
\end{tabular}

Obs: médias seguidas de letras iguais na coluna não diferem entre si, pelo teste de Tukey $(\mathrm{p}<0,05)$

* dados de plantas remanescentes em 1997, não incluídos nas análises estatísticas.
(Tabela 2). Iriarte-Martel et al. (1999) também não encontraram diferenças significativas, para massa fresca (peso) dos frutos, em função dos mesmos onze porta-enxertos estudados. Os valores encontrados de massa fresca dos frutos foram superiores ao valor médio relatado para o 'Tahiti' por Figueiredo (1991), que é de $70 \mathrm{~g}$ e, no presente trabalho, a média geral foi de $91 \mathrm{~g}$, valor também encontrado por Iriarte-Martel et al. (1999). A altura e diâmetro dos frutos mostraram-se dentro das faixas citadas por Donadio et al. (1995), $5,5 \mathrm{~cm}$ a $7 \mathrm{~cm} \mathrm{e} \mathrm{4,7} \mathrm{cm}$ a $6,3 \mathrm{~cm}$, para altura e diâmetro, respectivamente.

\section{Resistência à seca}

Os porta-enxertos comportaram-se de uma maneira semelhante em relação à resistência a seca, tendo sido o 'Orlando' considerado sensível nas três avaliações e 'Cravo' e 'Volkameriano Catania 2', resistentes (Tabela 3).

Na média das três avaliações, os porta-enxertos mais tolerantes à seca foram 'Cravo', 'Catania 2', sem diferirem entre si. 'Cravo' e 'Catania 2' diferiram dos demais porta-enxertos, exceto de 'Sunki', que, por sua vez, não diferiu de 'Swingle', 'Morton', 'Cleópatra' e trifoliata EEL, com resistência intermediária. Os porta-enxertos mais sensíveis foram : 'Orlando', 'Oneco', 'Batangas' e 'Caipira'.

\section{Desenvolvimento vegetativo das plantas}

O diâmetro médio das plantas foi influenciado pelos portaenxertos, com destaque para o 'Catania 2' que induziu os maiores

QUADRO 1 - Balanço hídrico climatológico - CAD 125mm - ( Thornthwaite \& Matter 1955) da Estação Experimetal de Citricultura de Bebedouro nos anos de 1991, 1994 e 1995 e dos municípios de Bebedouro-SP e Barretos-SP, segundo Ortolani et al. (1991). Bebedouro-SP, 2000.

\begin{tabular}{|c|c|c|c|c|c|c|c|c|c|c|c|c|}
\hline \multirow[b]{2}{*}{ Mês } & \multicolumn{4}{|c|}{1991} & \multicolumn{4}{|c|}{1994} & \multicolumn{4}{|c|}{1995} \\
\hline & ETP** & $\mathrm{P}$ & DEF & EXC & ETP & $\mathrm{P}$ & DEF & EXC & ETP & $\mathrm{P}$ & DEF & EXC \\
\hline Janeiro & 129,3 & 430,8 & 0,0 & 301,5 & 124,2 & 306,6 & 0,0 & 182,4 & 145,0 & 229,8 & 0,0 & $\overline{84,8}$ \\
\hline Fevereiro & 119,5 & 195,7 & 0,0 & 76,2 & 136,7 & 155,5 & 0,0 & 18,8 & 115,5 & 408,5 & 0,0 & 293,0 \\
\hline Março & 118,1 & 289,8 & 0,0 & 171,7 & 119,0 & 161,6 & 0,0 & 42,6 & 128,1 & 130,5 & 0,0 & 2,4 \\
\hline Abril & 96,2 & 192,4 & 0,0 & 96,2 & 99,1 & 34,3 & 14,2 & 0,0 & 89,4 & 37,5 & 9,4 & 0,0 \\
\hline Maio & 67,9 & 34,6 & 4,1 & 0,0 & 72,6 & 23,0 & 25,2 & 0,0 & 67,1 & 99,6 & 0,0 & 0,0 \\
\hline Junho & 62,3 & 1,4 & 24,0 & 0,0 & 53,1 & 13,6 & 25,9 & 0,0 & 52,7 & 10,8 & 9,1 & 0,0 \\
\hline Julho & 57,3 & 15,6 & 25,0 & 0,0 & 53,8 & 9,8 & 33,2 & 0,0 & 68,9 & 17,0 & 23,9 & 0,0 \\
\hline Agosto & 86,0 & 0,0 & 65,0 & 0,0 & 75,5 & 0,0 & 63,9 & 0,0 & 101,1 & 0,0 & 70,9 & 0,0 \\
\hline Setembro & 101,7 & 50,2 & 44,4 & 0,0 & 115,5 & 0,0 & 107,0 & 0,0 & 99,4 & 55,0 & 37,1 & 0,0 \\
\hline Outubro & 123,0 & 144,6 & 0,0 & 0,0 & 152,4 & 125,9 & 25,4 & 0,0 & 110,6 & 109,4 & 1,0 & 0,0 \\
\hline Novembro & 139,0 & 108,0 & 23,2 & 0,0 & 135,6 & 76,2 & 57,7 & 0,0 & 121,1 & 55,7 & 58,6 & 0,0 \\
\hline Dezembro & 136,6 & 252,5 & 0,0 & 18,7 & 142,7 & 240,7 & 0,0 & 0,0 & 133,6 & 269,6 & 0,0 & 21,0 \\
\hline$\overline{\mathrm{ANO}}$ & 1236,9 & 1715,6 & 185,5 & 664,3 & 1280,1 & 1146,9 & 352,5 & 243,8 & 1232,4 & 1423,4 & 210,1 & $\overline{401,1}$ \\
\hline $\begin{array}{r}\text { Bebedouro* } \\
(30 \text { anos })\end{array}$ & 1095,0 & 1325,0 & 91,0 & 321,0 & & & $\begin{array}{l}\text { rretos* } \\
\text { 0anos) }\end{array}$ & & 1140,0 & 1344,0 & 105,0 & $\overline{309,0}$ \\
\hline
\end{tabular}

*segundo Ortolani et al. (1991).

** $\mathrm{ETP}=$ evapotranspiração potencial; $\mathrm{P}=$ chuva; $\mathrm{Def}=$ deficiência hídrica; $\mathrm{Exc}=$ excedente hídrico 
valores, diferindo significativamente da maioria dos portaenxertos, exceto de 'Morton', 'Orlando' e trifoliata EEL (Tabela 4). A altura média das copas também foi influenciada pelos portaenxertos com 'Orlando' proporcionando o maior valor, diferindo de 'Batangas', 'Cleópatra' e 'Sunki', mas sem diferir dos demais. $\mathrm{Na}$ Tabela 4, foram inseridos os valores de altura, diâmetro e volume de copa referentes a uma única planta remanescente, em 1997, dos porta-enxertos 'Cravo' e 'Caipira DAC', apenas como indicadores do tamanho das copas com esses porta-enxertos. Notou-se má afinidade entre a copa e alguns porta-enxertos, principalmente com a 'Cleópatra', fato já relatado por Stenzel (1998), para o clone IAC-5 e por Stuchi et al.(dados não publicados), para a copa do clone 'Tahiti' premunizado oriundo do Centro Nacional de Pesquisa de Mandioca e Fruticultura Tropical, Cruz das Almas-BA, e cultivado em Bebedouro.

\section{CONCLUSÕES}

1) Podem ser recomendados como porta-enxertos alternativos ao limão-'Cravo', os seguintes, em ordem decrescente de produtividade: tangelo- 'Orlando', citrange-'Morton', citrumelo'Swingle', limão-'Volkameriano', trifoliata-'EEL', ressaltando que 'Orlando' é extremamente sensível à seca.

2) As menores produções são proporcionadas, ainda em ordem decrescente, pelas tangerinas-'Sunki', 'Cleópatra', Batangas' e 'Oneco' e pela laranja-'Caipira DAC', que não devem ser utilizadas como porta-enxertos para 'Tahiti'.

\section{AGRADECIMENTOS}

Os autores agradecem à FAPESP e ao CNPq, pelo financiamento parcial dessa pesquisa, e ao Fundo de Defesa da Citricultura - FUNDECITRUS, pelo empréstimo de veículos e pela colaboração financeira, bem como aos técnicos agrícolas Dimas Alves de Toledo e Luís Gustavo Parolin, pelo auxílio na condução e avaliação do experimento.

\section{REFERÊNCIAS BIBLIOGRÁFICAS}

DONADELLI, A., COELHO, P.J., SANTIAGO, M.M. D., NEGRI NETO, A. Valor da produção agropecuária do Estado de São
Paulo, 1996/97 e 1997/1998. Informações Econômicas, São Paulo, v.30, n.2, 2000.

DONADIO, L.C.; FIGUEIREDO, J.O. Hipertrofia do cálice, anomalia nova do limoeiro- Taiti. Campinas: IAC, 1972. 4p. (Circular, 14)

DONADIO, L.C.; FIGUEIREDO, J.O. de; PIO, R.M. Variedades cítricas brasileiras. Jaboticabal: FUNEP, 1995. 228 p.

FIGUEIREDO, J.O. de; RODRIGUEZ, O.; POMPEU JÚNIOR, J.; TEÓFILO SOBRINHO, J.; ABRAMIDES, E. Melhoramento do limoeiro - Taiti por seleção de clones . Bragantia, Campinas, v. 35 , p. $115-122,1976$.

FIGUEIREDO, J. O. de.Variedades copa de valor comercial. In: RODRIGUEZ, O.; VIÉGAS, F.; POMPEU JÚNIOR, J.; AMARO, A.A. coord. Citricultura brasileira. Campinas : Fundação Cargill, 1991 v.1, p. 228 - 264.

FIGUEIREDO, J.O.; DANADIO, L. C.; POMPEU JÚNIOR, J.; TEÓFILO SOBRINHO, J.; PIO, R. M.; VAZ FILHO, D.; STUCHI, E. S.; SEMPIONATO, O. R.; DOMINGUES, E. T. Comportamento de 11 porta-enxertos para o limão-Taiti na região de Bebedouro, SP. Revista Brasileira de Fruticultura, Cruz das Almas ,v.15, n.3, p. 345-351, 1996.

IRIARTE-MARTEL, J. H.; DONADIO, L. C.; FIGUEIREDO, J. O. de. Efeito de onze porta-enxertos sobre a qualidade dos frutos e desenvolvimento das plantas da lima ácida 'Tahiti'. Revista Brasileira de Fruticultura, Jaboticabal, v.21, n.2, p. 235 -238, 1999.

RODRIGUEZ, O.; FIGUEIREDO, J. O.; POMPEU JÚNIOR, J.; TEÓFILO SOBRINHO, J. Tristeza no limoeiro tahiti- Seleção de clones. In: CONGRESSO BRASILEIRO DE FRUTICULTURA, 2. , 1973, Viçosa. Anais... Viçosa: SBF, 1973. v.1, p. 367-379.

STENZEL, N. M. C. Comportamento de porta-enxertos para a lima ácida 'Tahiti' no Estado do Paraná . In: CONGRESSO BRASILEIRO DE FRUTICULTURA, 15., Poços de Caldas. Resumos... Poços de Caldas:Sociedade Brasileira de Fruticultura, 1998. p. 289. 